\title{
Effects of Feeding Fermented Fish on Egg Cholesterol Content in Hens
}

\begin{abstract}
This study was conducted to investigate the effects of feeding fermented fish (FF) to layers on laying performance, and polyunsaturated fatty acid and cholesterol levels in eggs and plasma. A total of 96, 13-week-old Babcock B380 pullets were used in this study. They were randomly assigned to four numerically equal groups with eight replicates per treatment, three birds per replicate. All the birds were housed in individual cages. The dietary treatments were: Control diet, without FF; FF3 diet containing 3\% (w/w) FF, FF6 diet containing 6\% (w/w) FF and FF9 diet containing 9\% (w/w) FF. The study was carried out for 16 weeks inclusive of two weeks of adjustment. Weekly feed intake and egg production were recorded. Blood plasma cholesterol and fatty acid profiles were assayed at the end of the experiment. FF did not enhance $(\mathrm{P}>0.05)$ egg mass but $(\mathrm{P}<0.05)$ decreased egg weight slightly. However, egg yolk cholesterol and plasma cholesterol concentrations were reduced $(\mathrm{P}<0.05)$ by FF. The $\mathrm{n}-$ 6:n-3 fatty acids ratio in the egg yolk (Control $=7.9$, FF9 $=6.2)$ and plasma $($ Control $=10.6$, FF9 = 6.2) were decreased by feeding FF. Moreover, FF was able to increase $(\mathrm{P}<0.05)$ the docosahexaenoic acid (DHA) concentrations in egg yolk and plasma. In conclusion, this study demonstrated that FF increased DHA and reduced egg yolk cholesterol in poultry eggs.
\end{abstract}

Keyword: Egg production, Egg quality, Fatty acids, Fermented fish, Layer 